\title{
Joint Estimation of Propagation Delay Dispersion and Time of Arrival in a 40-nm CMOS Comparator Bank for Time-Based Receivers
}

\author{
Tom Redant, Student Member, IEEE, and Wim Dehaene, Senior Member, IEEE
}

\begin{abstract}
This brief presents an approach to compensate time-of-arrival (ToA) estimation errors caused by the effect of signal depending propagation delays in receivers for ranging applications. As a straightforward reasoning, reducing the ToA error results in increased power consumption in the analog-frontend blocks of such receivers. In the proposed system topology, the asynchronous receiver's analog-front-end blocks contain an array of identical continuous-time comparators, which makes it suitable for a joint modeling approach and today's low-supply voltages. Attention is paid to tuning the nonstrobed comparator topology to make it suitable for this joint modeling. Using the comparator model in a least-squares-based algorithmic approach can reduce the ToA error (accuracy improvement) to nearly zero. A ToA systematic error reduction of 28 is faced for the ToA figure. Furthermore, energy consumption is more related to the activity of the ranging events, which makes this an interesting approach for ranging measurement rates of less than $1 \mathrm{MHz}$.
\end{abstract}

Index Terms-Asynchronous digitization, comparator bank, propagation delay, time of arrival (ToA).

\section{INTRODUCTION}

$\mathbf{T}$ IME-OF-ARRIVAL (ToA) estimation is a core principle in wireless ranging applications. To achieve low-power receiver implementations, it is beneficial to use an asynchronousdigitization approach based on low standby-power consuming time-to-digital converters (TDCs) [1], [2]. Fig. 1 shows the receiver's block diagram for such a low-power ToA-based ranging application. It will be the application-of-interest for this brief. In this application, the ToA is estimated by identifying an increase in the received signal's envelope while using multiple asynchronous digitizers as seen in [3] and [4]. There is a fundamental difference between classic synchronous-digitization methods and this asynchronous-digitization principle, as shown in the figure. In asynchronous digitization, digital values are generated at a level-crossing event, providing a better digital

Manuscript received August 28, 2012; revised November 3, 2012; accepted December 9, 2012. Date of publication March 7, 2013; date of current version March 13, 2013. This work was supported by the Flemish agency for Innovation by Science and Technology and Essensium NV. This brief was recommended by Associate Editor E. Bonizzoni.

T. Redant is with the Microelectronics and Sensor Laboratory (MICAS), Department of Electrical Engineering (ESAT), Catholic University of Leuven (K.U. Leuven), 3001 Heverlee, Belgium (e-mail: tom.redant@esat.kuleuven.be).

W. Dehaene is with MICAS, ESAT, Catholic University of Leuven (K.U. Leuven), 3001 Heverlee, Belgium, and also with the Interuniversity Micro-Electronics Centre, 3001 Leuven, Belgium (e-mail: wim.dehaene@esat. kuleuven.be)

Color versions of one or more of the figures in this brief are available online at http://ieeexplore.ieee.org.

Digital Object Identifier 10.1109/TCSII.2012.2235736

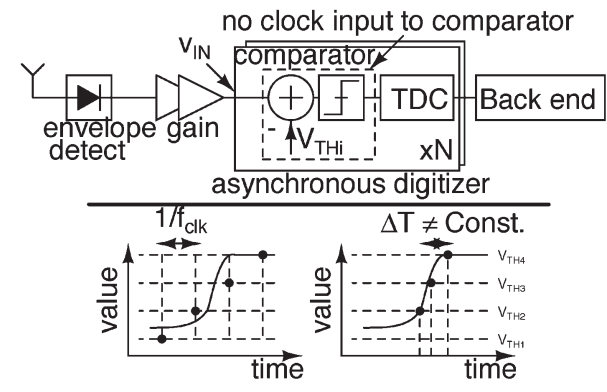

Fig. 1. (Top) Receiver topology of interest. (Bottom Left) Classic synchronous digitization. (Bottom Right) Low-power highly precise asynchronous digitization based on TDCs.

representation of the time domain compared to a synchronous digitization. Nonstrobed comparators need to be applied for this topology. Despite the sporadic activity of these comparators, they consume a significant amount of standby power when they need to be fast. In the quest for a low-power receiver implementation, comparator power consumption should be kept as low as possible, implying finite comparator response times. These response times (the propagation delays) are a complex function of the comparator topology, its biasing, and the signal shape.

Thus, the comparator propagation delay should be independent of the exact shape of the signal or the comparator. Unfortunately, no nonstrobed (continuous-time) comparator topology, consuming a finite amount of power, is able to realize this independence. This brief introduces a joint application and signal-aware approach to deal with these input signal depending comparator propagation delays and to compensate for them to remove its structural error effect on the ToA metric. That is achieved with focus on low-voltage technologies. Asynchronous digitization and DSP approaches [5] are widely discussed. On the other hand, the power consumption associated with generating the required asynchronous digital events, as one of their biggest drawbacks, is often disregarded. This brief will therefore try to close this gap. The ultimate incentive for this brief is to shift a typically high amount of continuous analog comparator power toward activity-related power in the digital domain. This means that the approach is always beneficial for sporadic ranging events. The proposed methodology can be applied to many more domains where comparator-bank propagation delays need to be compensated, as is the case for stochastic TDCs [6].

It should be noted that this brief does not claim to provide optimal comparator threshold value selection; the literature 
provides answers to that [3], [7]. Additionally, this brief does not motivate the need for multiple comparators for ToA estimation since this is already available in the literature [3], [8].

Section II situates the signal structure for the discussed system and the comparator topology. Next, Section III introduces the level setting of the comparator topology and the problem of the input signal depending comparator propagation delays. Section IV shows how to deal with this imperfection based on both theoretical and mathematical approach, and a numerical example that shows how to make a decision about comparator sizing. Section $\mathrm{V}$ will show the results for the final comparator bank in 40-nm CMOS and an iterative implementation of the algorithm and power figures. Conclusions are drawn in Section VI.

\section{SyStem OF INTEREST}

As mentioned in the Introduction, this brief focuses on the elimination of variable propagation delays for comparators in an asynchronous receiver for a ranging application (Fig. 1). The main focus in this ranging application will be the estimation of the parameter $t_{\text {toa }}$, the ToA of the signal. The received signal after envelope detection (Fig. 1) is defined as $v_{\mathrm{IN}}$, as shown in (1). It is a function of time $t_{\text {toa }}$ and $M$ additional parameters $\varphi_{i}$, of which the values are not known by the receiver. These parameters $\varphi_{i}$ influence the ToA estimation process and should therefore be included in further derivations

$$
v_{\mathrm{IN}}\left(t, t_{\mathrm{toa}}, \varphi_{0}, \ldots, \varphi_{\mathrm{M}-1}\right)
$$

These additional parameters can be a varying channel bandwidth $B$, the signal's amplitude $A$, and additional wireless channel model parameters. The remainder of this section will introduce the considered comparator for the estimation strategy as presented in this brief.

\section{A. Comparator-Bank Topology Selection}

Fig. 2 shows the comparator topology of interest. The amount of stacked transistors is kept low for low-supply operation ( $0.9 \mathrm{~V}$ in $40-\mathrm{nm}$ CMOS). The active devices are put in an OTA configuration having an NMOS input differential pair, loaded with PMOS current sources and diode-connected PMOS transistors. The diode-connected PMOS transistors prevent the input differential pair transistors from going out of the saturation region. No feedback is applied to the comparator load transistors. Moreover, feedback implies hysteresis, introducing additional modeling difficulties during the estimation of comparator-propagation dispersion. $C_{\mathrm{l}}$ is the load capacitor. The capacitive-resistive input network provides AC coupling of the input signal and level setting. It will be considered later in this text.

\section{COMPARATOR-PROPAGATION-DELAY DISPERSION}

The applied comparator topology will, like any other comparator, suffer from input signal depending comparator propagation delays. The effect of the input signal is twofold.

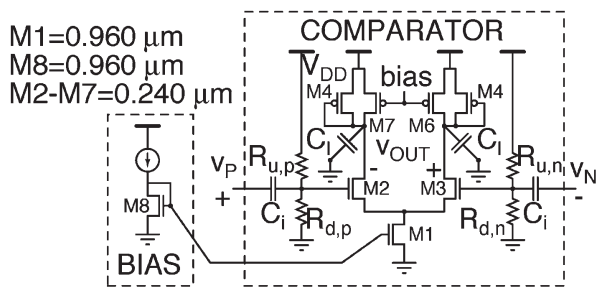

Fig. 2. Proposed comparator cell structure.

First, comparators face a threshold value depending propagation delay. Additionally, they face an input signal depending propagation delay. In this section, at first, a threshold levelsetting network is added to the comparator in such a way that the comparator will be functional over a high range of threshold values, reducing the propagation delay's sensitivity to the threshold value. Second, a mathematical description of the comparator-propagation-delay dispersion is provided.

\section{A. Circuit-Based Propagation-Delay-Dispersion Suppression}

Consider the comparator fires at an extremely high or low comparator threshold value. Then, while firing, no optimal gate voltages are present on the input differential pair. Transistors M2 and M3 can be pinched-off or tend to go out of their saturation region, resulting in a poor propagation delay. Thus, due to the low-supply voltage, no significant input range for the comparator threshold values is available to the designer. That is why a passive input biasing network is applied serving as its level setting. The resistive dividers $\left(R_{\mathrm{u}, \mathrm{p}}, R_{\mathrm{d}, \mathrm{p}}\right)$ and $\left(R_{\mathrm{u}, \mathrm{p}}, R_{\mathrm{d}, \mathrm{p}}\right)$ are sized to set all comparators in the comparator bank to fire at a common mode of $0.6 \mathrm{~V}$, independent of the comparator threshold value. The $C_{\mathrm{i}}-R_{\mathrm{i} / / \text {-network }\left(R_{\mathrm{i} / /}=\right.}$ $\left.\left(R_{\mathrm{u}} \cdot R_{\mathrm{d}}\right) /\left(R_{\mathrm{u}}+R_{\mathrm{d}}\right)\right)$ does this by modifying the differential gate voltage of M2-M3 of the comparator at DC. However, in a small interval after $t=t_{\text {toa }}$, the output does not see an impact of the $C_{\mathrm{i}}-R_{\mathrm{i} / / \text {-network apart from its level-setting }}$ behavior. One should size the passives in such a way that the following condition is satisfied for the zero frequency: $1 /\left[2 \pi \cdot R_{\mathrm{i} / /} \cdot C_{\mathrm{i}}\right] \ll f_{\mathrm{r}}$, with $f_{\mathrm{r}}$ as the ranging measurement rate. Of course, $f_{\mathrm{r}} \ll B$. The zero frequency is set at $0.1 \mathrm{MHz}$. For the sake of having a reduced biasing power $(10 \mu \mathrm{W}$ per resistive divider), $C_{\mathrm{i}}$ upsizing is preferred. The latter is also beneficial for the amount of integrated supply noise on the input differential nodes, which is $k T / C_{i}(k=$ Boltzmann constant, $T=$ the circuit temperature). The modeling section will omit the $C_{\mathrm{i}}-R_{\mathrm{i} / / \text {-network since it has no influence on short-time }}$ transients.

The comparator is now suited for working at high comparator threshold values. As an interesting consequence, all comparators in the bank are identical in terms of their active devices, reducing circuit design and layout overhead. Moreover, the same model could be used for all of them in the next sections.

\section{B. Modeling the Comparator Propagation Delay}

The load of the comparator of Fig. 2 is a single PMOS current source and a diode-connected PMOS. The latter is to keep the input transistors in its saturation region when the comparator 
has made a decision by not allowing the output voltage to go below $V_{\mathrm{DD}} / 2$. A differential output swing of $V_{\mathrm{DD}}=0.9 \mathrm{~V}$ is thus enabled. Under the assumption that the input differential pair has a significant transconductance $g_{\mathrm{m}}$, one can use the small-signal model to calculate the propagation delay between the signals $v_{\mathrm{IN}}=v_{\mathrm{P}}-v_{\mathrm{N}}$ and $v_{\mathrm{OUT}}$. As stated earlier, this is true for signals that have their frequency content beyond the zero frequency of the input level shift network $C_{\mathrm{i}}-R_{\mathrm{i} / /}$ for which the system is designed.

Assume that the small-signal input can be linearly approximated as $v_{\text {in }}(t)=\alpha_{i} \cdot\left(t-t_{\text {toa }}\right)$ while crossing the comparator level $V_{\mathrm{TH}_{i}}<A$, with $\alpha_{i}[V / s]$ as the input signal's slope during comparator output node switching. Equation (2) shows the relationship of the propagation delay $\Delta t_{\mathrm{p}_{i}}$, the output current $i_{\text {out }}(t)$, and the output voltage transition of a value of $V_{\mathrm{DD}}$

$$
\begin{aligned}
\int_{t_{\mathrm{toa}}}^{t_{\mathrm{toa}}+\Delta t_{\mathrm{p}_{i}}} \frac{i_{\mathrm{out}}(t)}{C_{\mathrm{l}}} d t & =\int_{t_{\mathrm{toa}}}^{t_{\mathrm{toa}}+\Delta t_{\mathrm{p}_{i}}} \frac{g_{\mathrm{m}} \cdot v_{\mathrm{IN}}(t)}{C_{\mathrm{l}}} d t \\
& =\left[g_{\mathrm{m}} \cdot \alpha_{i} \cdot \Delta t_{\mathrm{p}_{i}}^{2}\right] /\left[2 \cdot C_{\mathrm{l}}\right]=V_{\mathrm{DD}} .
\end{aligned}
$$

$C_{1}=1 \mathrm{fF}$ throughout this brief. The propagation delay can be extracted out of (2)

$$
\Delta t_{\mathrm{p}_{i}}=\sqrt{2 \cdot \frac{V D D \cdot C_{\mathrm{l}}}{\alpha_{i} \cdot g_{\mathrm{m}}}} .
$$

This would suggest that the ToA estimation's accuracy would improve by a factor of $\sqrt{g}_{\mathrm{m}}$ as a function of $g_{\mathrm{m}}$, stating that only when having an infinite amount of power, the ToA accuracy will go to zero. One should be aware of the fact that $\alpha_{i}$ is a function of the comparator index $i$ and does strongly depend on the comparator's input signal's shape. This $\alpha_{i}$ is the source of the unwanted comparator-propagation-delay dispersion. The next section will show how to compensate for this in the considered ranging system.

\section{REMOVING REMAINING \\ COMPARATOR-PROPAGATION-DELAY DISPERSION}

In this section, a theoretical framework for the estimation technique is introduced based on closed-form mathematical expressions for the comparator propagation delay resulting in a set of equations. A numerical example is provided to support the mathematical findings.

\section{A. Theoretical Approach}

1) General Theory: As already shortly introduced in [2], an estimation of the comparator propagation delay is possible for asynchronous A/Ds. The equations in [2] will be considered as a starting point for the derivation provided in this brief. Assume that a bank of $N$ comparators have a threshold voltage $V_{\mathrm{TH}_{i}}$, produce an event at $t_{i}$, and then one has the following set of equations for propagation delay estimation

$$
\begin{cases}\Delta t_{\mathrm{p}_{i}}=g\left(V_{\mathrm{TH}_{i}}, \varphi_{0}, \ldots \varphi_{\mathrm{M}-1}\right) & \forall 0 \leq i<N \\ V_{\mathrm{TH}_{i}}=v_{\mathrm{IN}}\left(t_{i}-\Delta t_{\mathrm{p}_{i}}, t_{\mathrm{toa}}, \varphi_{0}, \ldots, \ldots \varphi_{M-1}\right) & \forall 0 \leq i<N .\end{cases}
$$

This is a set of $2 N$ equations in $M+N+1$ unknowns $\left\{\Delta t_{\mathrm{p}_{0}}, \ldots, \Delta t_{\mathrm{p}_{\mathrm{N}-1}}, \varphi_{0}, \ldots, \varphi_{\mathrm{M}-1}, t_{\text {toa }}\right\}$. If $N=M+1$, then this is an ordinary set of nonlinear equations, which can be solved by using the Newton-Raphson method. If $N>M+1$, then this is a nonlinear least squares problem, which can be solved by the Gauss-Newton algorithm. The first $N$ equations model the comparator propagation delay as a closedform function $g$ of the comparator threshold and the parameter set $\left\{\varphi_{0}, \ldots, \varphi_{\mathrm{M}-1}\right\}$. The final $N$ equations relate the comparator threshold values to the signal's crossing event of the comparators.

2) Application-Specific Theory: The range measurements are carried out on a signal as defined in (5). One can, e.g., assume that $\mathrm{B}$ and $\mathrm{A}$ are unknown parameters to the receiver. This elaborates (1) to $v_{\mathrm{IN}}\left(t, t_{\text {toa }}, B, A\right)$

$$
v_{\mathrm{IN}}\left(t, t_{\mathrm{toa}}, B, A\right)=v_{\mathrm{P}}-v_{\mathrm{N}}=A \cdot\left[1-e^{-2 \pi \cdot B \cdot\left(t-t_{\mathrm{toa}}\right)}\right] .
$$

The signal-of-interest is a $t_{\text {toa }}$ time-shifted filtered edge function. The filtering models the channel, which is assumed to be a first-order low-pass filter with cutoff frequency $B$ at the baseband. The signal equals the rising edge of a block-pulse traveling through a band-limited channel and will be the signal for range measurements. For the signal-of-interest defined by (5), it can be shown that $\alpha_{i}$ is a function of $V_{\mathrm{TH}_{i}}$

$$
\alpha_{i}=2 \pi \cdot B \cdot\left(A-V_{\mathrm{TH}_{i}}\right) .
$$

That would mean for the comparator propagation delay

$$
\begin{aligned}
\Delta t_{\mathrm{p}_{i}} & =\sqrt{\frac{2 \cdot V D D \cdot C_{\mathrm{l}}}{2 \pi \cdot B \cdot\left(A-V_{\mathrm{TH}_{i}}\right) \cdot g_{\mathrm{m}}}} \\
& =g\left(V_{\mathrm{TH}_{i}}, B, A\right) .
\end{aligned}
$$

This states that the system faces input signal depending propagation delays, since the $\Delta t_{\mathrm{p}_{i}}$ is a function $g$ of the input signal's parameter set $\{A, B\}$.

Having (5), $M=2$ and therefore at least three comparators $(N=3)$ are needed to solve the problem. One gets a root finding problem $F: \mathbb{R}^{6} \rightarrow \mathbb{R}^{6}$, implemented by a set of nonlinear equations in the unknown $X=\left[\Delta t_{\mathrm{p}_{0}}, \Delta t_{\mathrm{p}_{1}}, \Delta t_{\mathrm{p}_{2}}, B, A\right.$, $\left.t_{\text {toa }}\right]^{\top}$. The vector $F=\left[f_{0}(X) \ldots f_{5}(X)\right]^{\top}$ of equations according to (4) will have the following components $\forall 0 \leq i<2$ :

$$
\begin{gathered}
f_{i}(X) \equiv \Delta t_{\mathrm{p}_{i}}-\sqrt{2 \cdot \frac{V D D \cdot C_{\mathrm{l}}}{2 \pi \cdot B \cdot\left(A-V_{\mathrm{TH}_{i}}\right) \cdot g_{\mathrm{m}}}}=0 \\
\left.f_{i+N}(X) \equiv V_{\mathrm{TH}_{i}}-A \cdot\left(1-e^{-2 \pi \cdot B \cdot\left(t_{i}-\Delta t_{\mathrm{p}_{i}}-t_{\mathrm{toa}}\right.}\right)\right)=0 .
\end{gathered}
$$

Based on this, the Jacobian matrix $J_{F} \in \mathbb{R}^{6 \times 6}$ can be constructed. Newton-Raphson method is used for solving the set of equations based on this matrix.

An important property of the solution of a set of equations is its sensitivity to noise, which is circuit noise, wireless channel noise, quantization noise, or mathematical rounding. This manifests as a perturbation in the voltage domain (with standard 
deviation $\sigma_{\mathrm{v}}$ ), modeled as a contribution in the right-hand sides of $f_{0}(X)$ to $f_{N-1}(X)$ in (8). It can also manifest in the time domain (with standard deviation $\sigma_{\mathrm{t}}$ ), modeled as a contribution in the right-hand sides of $f_{N}(X)$ to $f_{2 \cdot N-1}(X)$ in (8). The latter can be a result of the finite time resolution of the eventdetector (e.g., the TDC). Note that nonidealities different from noise such as comparator input referred offset due to silicon fabrication variability will result in a deterministic $V_{\mathrm{TH}_{i}}$ shift. This can be measured and compensated by the technique as well and therefore will not be considered in this brief as part of the main problem.

Susceptibility of the solution to noise perturbations is usually verified by the condition number of the matrix $J_{F}$. The condition number will state that the problem is illconditioned. However, one is not interested in the complete multidimensional stochastic solution vector of (4), which is $\left[\Delta \mathrm{t}_{\mathrm{p}_{0}}, \Delta \mathrm{t}_{\mathrm{p}_{1}}, \Delta \mathrm{t}_{\mathrm{p}_{2}}, \mathrm{~B}, \mathbf{A}, \hat{t_{\mathrm{toa}}}\right] . \mathrm{t}_{\mathrm{toa}}$ is the only unknown of interest. Therefore, instead of using the condition number, sensitivity to noise is analyzed based on the squared addition of all contributing noise sources for the $\mathbf{t}_{\mathrm{toa}}$. One can assume that there is no correlation between the different noise contributors. In a real system, there is a correlation due to the band-limited behavior, although this assumption does not jeopardize the technique's applicability. The precision (in terms of the standard deviation $\sigma_{t_{\text {toa }}}$ ) of the $\hat{t}_{\text {toa }}-t_{\text {toa }}$ when having noise is

$$
\sigma_{t_{\text {toa }}}=\left\|\left[\left(J_{F}^{-1}\right)_{\{6,:\}}\right] \odot\left[\sigma_{\mathrm{v}} \sigma_{\mathrm{t}} \sigma_{\mathrm{v}} \sigma_{\mathrm{t}} \sigma_{\mathrm{v}} \sigma_{\mathrm{t}}\right]\right\|_{2} .
$$

$\odot$ is the elementwise vector product. \|\|$_{2}$ the vector's 2-norm and $X_{\{6,:\}}$ stands for extracting the 6th row of a matrix. Accuracy (the mean error on the ToA) can be reduced to virtually zero since the zero-mean Gaussian noise contributions have a linear effect on the $\hat{t}_{\text {toa }}$. This means that the error on $\mathbf{t}_{\text {toa }}-t_{\text {toa }}$ is also zero mean. Thus only the precision $\sigma_{t_{\text {toa }}}$ needs to be verified while evaluating the performance of the technique. Equation (9) defines an important figure that shows the performance of the comparator-propagation-delaydispersion estimation. A numerical example will illustrate the strength of this equation in the next section.

\section{B. Numerical Example}

As a numerical example to state the sensitivity to noise, (9) is evaluated for $\sigma_{t}=10 \mathrm{ps}, \sigma_{v}=2 \mathrm{mV}$, and $V_{\mathrm{TH}_{0}} \ldots V_{\mathrm{TH}_{2}}=$ [5 $\left.\begin{array}{lll}50 & 100 & 150\end{array}\right] \mathrm{mV}$. Results for the evaluation of this numerical example are shown in Fig. 3. The figure shows that the ToA stochastic variable $\mathbf{t}_{\text {toa }}$ is not highly sensitive to noise whenever facing lower comparator transconductances. The comparator propagation delay faced at the $200-\mathrm{mV}$ level for the signal of interest is initially seen around 100 ps and is now compensated, moreover, at a good precision. Systematic deviations of the comparator propagation delay are now compensated. The problem is translated to a zero-mean noise problem and can thus be averaged over multiple range measurements. That is an important conclusion. One can compare this to dithering in the field of audio and image processing, in which unwanted systematic distortion is transformed into less disturbing zero-

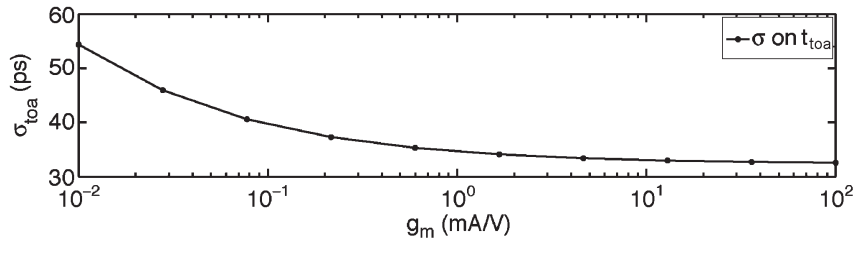

Fig. 3. Precision on the ToA parameter as a function of the transconductance $g_{\mathrm{m}}$. The ToA parameter does not suffer too much from a low transconductance.

mean perturbations. This example also states that estimation is always possible, no matter how sloppy or low power the comparator is, but of course this increase of accuracy comes at a precision cost (increased $\sigma_{\text {toa }}$ ).

This mathematical modeling and numerical example provides a good strategy in the process of finding the $g_{\mathrm{m}}$ value one should design for. For example, in this three-comparator example, a good choice for $g_{\mathrm{m}}$ will be $g_{\mathrm{m}}=1 \mathrm{~mA} / \mathrm{V}$ since higher $g_{\mathrm{m}}$ values do not provide a significant reduction of $\sigma_{\text {toa }}$. $g_{\mathrm{m}}$ fixes the comparator's sizing.

\section{REAL-WORLD APPLICATION}

The presented approach is now applied to a real ranging application receiver that tries to find the ToA in the presence of channel noise and a known received signal (5) with unknown amplitude $A$ and $t_{\text {toa }}$. The bandwidth $B$ is fixed for the ranging system. This is a good assumption since it is determined by the accurate and precise nature of the resonant $\mathrm{L}, \mathrm{C}$ and $\mathrm{R}$ passives in $\mathrm{RF}$ front-ends. It is taken at $250 \mathrm{MHz}$.

\section{A. Comparator Model}

The previous section assumed a perfect knowledge of the comparator-propagation-delay behavior (the $g$-function). Moreover, it started from a small-signal model. Now, the proposed methodology will be tested against the real comparator behavior for the topology in Fig. 2 in 40-nm CMOS, as it will be in the application. Fig. 2 provides the width sizings of the MOSFETs. Lengths are chosen as 40-nm minimal. The DC power, consumed by a single comparator, is $58.5 \mu \mathrm{W}$.

Although simplified comparator models are available in the literature [9], this brief uses HSPICE simulation data to create a lookup table as a model. Together with the thresholds $V_{T H_{i}}$, this is the only dataset that needs to be measured or simulated to come to a working algorithm. The lookup table is visualized in Fig. 4. It contains the comparator propagation delays (the $g$-function) for the signal $v_{\text {IN }}$. For the creation of this table, the input signal $v_{\text {IN }}$ is assumed to hard clip at the supply $V_{\mathrm{dd}}$ and the ground. The plot shows a variable comparator propagation delay. The main tendency is the higher the threshold value, the slower the comparator. The comparator propagation delay is about $140 \mathrm{ps}$ for $V_{\mathrm{TH}}=300 \mathrm{mV}$ and $A=500 \mathrm{mV}$. Accuracy is thus limited to about $140 \mathrm{ps}$. The domain of the plot is limited to $V_{\mathrm{TH}}<A$. That makes sense since this is a fundamental condition for a comparator event. Fig. 4 is used for modeling an eight-way comparator bank, triggering at levels (in millivolts) $\{8,49.7,91.4,133,174.9,216.6,258.3,300\}$ and $A=500 \mathrm{mV}$. 


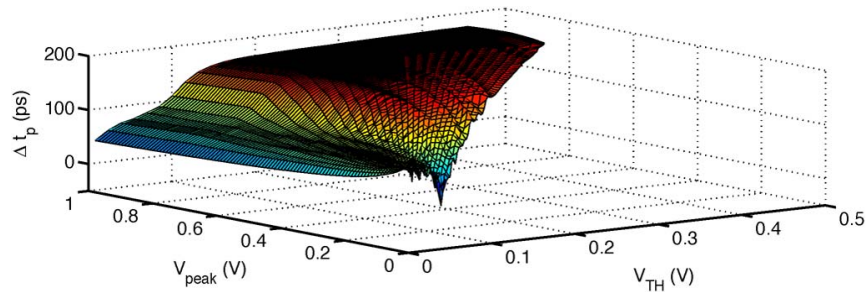

Fig. 4. Comparator $\Delta t_{\mathrm{p}_{i}}$ as a function of $V_{\mathrm{TH}}$ and $A$. After applying the $C_{\mathrm{i}}-R_{\mathrm{i} / /}$ network, a significant dispersion is still noticed.

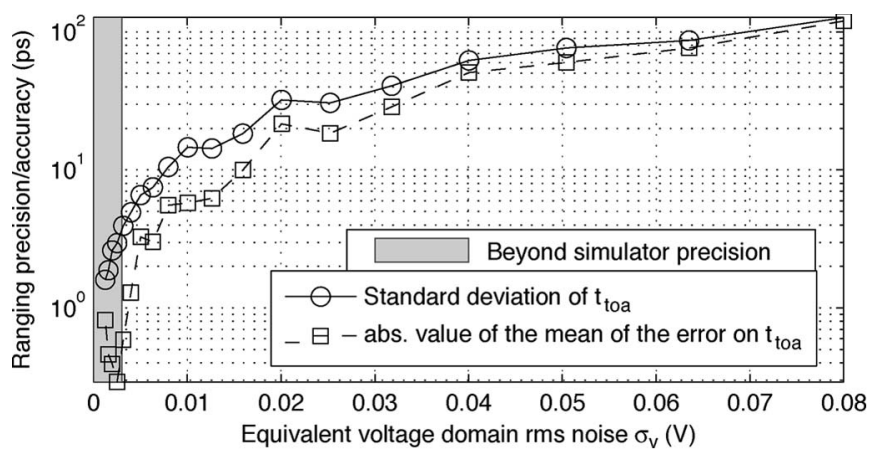

Fig. 5. Performance plot of the comparator-propagation-delay-dispersionestimation technique. The simulation took 100 noise samples per $\sigma_{\mathrm{v}}$ value.

\section{B. The Algorithm}

$J_{F}$ is constructed out of (4) for the unknown $X=\left[\Delta t_{\mathrm{p}_{0}}, \ldots\right.$ $\left.\Delta t_{\mathrm{p}_{7}}, A, t_{\mathrm{toa}}\right]^{\top}$. The Gauss-Newton algorithm contains iterations of the form

$$
\begin{aligned}
X^{(k+1)} & =X^{(k)}+\Delta^{(k)} \quad \text { with } \\
\Delta^{(k)} & =\left.\left(J_{F}^{\top} \cdot J_{F}\right)^{-1} \cdot\left(-J_{F}^{\top}\right) \cdot F\right|_{X=X^{(k)}} .
\end{aligned}
$$

$X^{(k)}$ is the approximation of the unknown $X$ at the $k^{\text {th }}$ iteration. The values $\Delta t_{\mathrm{p}_{\mathrm{i}}}^{(0)}=100 \mathrm{ps}, t_{\mathrm{toa}}^{(0)}=t_{0}$, and $A^{(0)}=$ $\max _{\mathrm{i}} V_{\mathrm{TH}_{i}} / 0.9$ are taken as initial values.

The number of iterations is fixed at 10 since convergence is not significant anymore beyond this value. Thanks to the sparse $J_{F}$ matrix, only about 300 multiplications and additions, 16 exponentials, and 1 matrix inverse are executed per iteration. This can be carried out on a single multiplication and addition structure due to the sporadic nature of ranging events. Results for different values of $\sigma_{\mathrm{v}}$ are shown in Fig. 5. One can see that accuracy is improved from 140 to 5 ps (gain of 28) even when facing an RMS voltage noise of $10 \mathrm{mV}_{\mathrm{rms}}$. One can notice that the precision is also high $\left(\sigma_{\text {ToA }} \approx 10\right.$ ps around $10-\mathrm{mV}$ RMS comparator threshold noise). Reducing the RMS noise more to zero improves precision and accuracy even more thanks to this technique, which implies a significant improvement in the performance compared to the noncompensated case. Extensive simulations show that at least a 14-bit fractional representation is needed to consider rounding errors as negligible.

In order to come to a power figure, both multiplications and additions are taken as the major power contributors. Moreover, in a naïve multiplier implementation, it is a good practice to consider one multiplication as equivalent to 14 additions. HSPICE simulations show that one ripple-carry 14-bit addition consumes about $90 \mathrm{fJ}$ in 40-nm CMOS. Based on the computational complexity, one range measurement consumes $4.05 \mathrm{~nJ}$ of digital processing power. Even if the range measurement rate goes up to $f_{r}=1 \mathrm{MHz}$, power is limited to about $4 \mathrm{~mW}$. Analog domain comparator optimization [10], on the other hand, will only provide an accuracy gain of 12 at the cost of adding multiple comparator stages, which brings the stand-by power for one single comparator also in the milliwatt range [10]. Digital delay line-assisted compensation [11] provides only a gain of 6.

\section{CONCLUSION}

This brief shows a technique jointly obtaining a ToA estimate and postcompensating for comparator-bank propagation delay dispersion due to slow low-power nonstrobed comparators in asynchronous low-power ranging receivers. The method presented in this brief enables shifting standby power toward activity-related power, which is beneficial for sporadic event ranging receivers $\left(f_{r}<1 \mathrm{MHz}\right)$. A comparator for this application is introduced. To extend the range of the comparator bank, first, a level setting based on a passive network is applied. The problem is formulated as a nonlinear least squares set of equations. The joint approach enables direct evaluation of the refined ToA estimate. An implementation in an asynchronous receiver's back-end enables a picosecond precision for the ToA for the 40-nm CMOS comparator bank as presented here.

\section{REFERENCES}

[1] J. Nissinen, I. Nissinen, and J. Kostamovaara, "Integrated receiver including both receiver channel and TDC for a pulsed time-of-flight laser rangefinder with cm-level accuracy," IEEE J. Solid-State Circuits, vol. 44, no. 5, pp. 1486-1497, May 2009.

[2] T. Redant, F. Stubbe, and W. Dehaene, "A low power time-of-arrival ranging front end based on a 8-channel $2.2 \mathrm{mw}, 53 \mathrm{ps}$ single-shotprecision time-to-digital converter," in Proc. IEEE A-SSCC, Nov. 2011, pp. 321-324.

[3] T. Redant, J. Daniels, M. S. J. Steyaert, and W. Dehaene, "Multiple event time-to-digital conversion-based pulse digitization for a $250 \mathrm{MHz}$ pulse radio ranging application," IEEE Trans. Circuits Syst. I, Reg. Papers, vol. 58, no. 11, pp. 2614-2622, Nov. 2011.

[4] J.-P. Jansson, V. Koskinen, A. Mantyniemi, and J. Kostamovaara, "A multichannel high-precision CMOS time-to-digital converter for laserscanner-based perception systems," IEEE Trans. Instrum. Meas., vol. 61, no. 9, pp. 2581-2590, Sep. 2012.

[5] Y. Tsividis, "Event-driven data acquisition and digital signal processingA tutorial," IEEE Trans. Circuits Syst. II, Exp. Briefs, vol. 57, no. 8, pp. 577-581, Aug. 2010.

[6] V. Kratyuk, P. K. Hanumolu, K. Ok, U.-K. Moon, and K. Mayaram, "A digital PLL with a stochastic time-to-digital converter," IEEE Trans. Circuits Syst. I, Reg. Papers, vol. 56, no. 8, pp. 1612-1621, Aug. 2009.

[7] K. Kozmin, J. Johansson, and J. Delsing, "Level-crossing ADC performance evaluation toward ultrasound application," IEEE Trans. Circuits Syst. I, Reg. Papers, vol. 56, no. 8, pp. 1708-1719, Aug. 2009.

[8] I. Nissinen and J. Kostamovaara, "A 2-channel CMOS time-to-digital converter for time-of-flight laser rangefinding," in Proc. IEEE I2MTC, May 2009, pp. 1647-1651.

[9] R. V. D. Plassche, CMOS Integrated Analog-to-Digital and Digital-toAnalog Converters, 2nd ed. Boston, MA: Kluwer, 2003.

[10] K. Kozmin, J. Johansson, and J. Delsing, "A low power, propagation delay stable, continuous-time comparator," in Proc. Norchip Conf., Nov. 2004, pp. 261-264.

[11] H. Yu, R. Miyaoka, and T. Lewellen, "Investigation of delay compensation circuit techniques to reduce timing walk," in Conf. Rec. IEEE Nucl. Sci. Symp., 1998, vol. 1, pp. 412-416. 